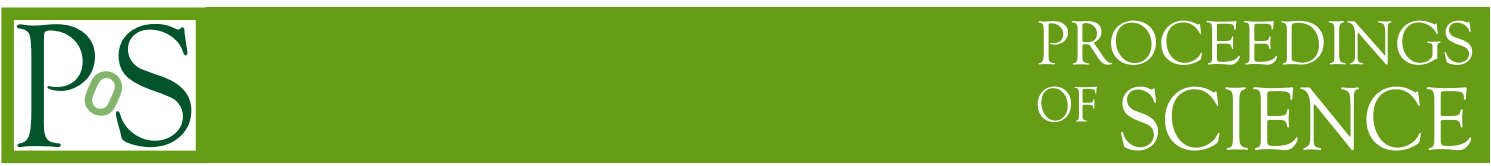

\title{
The influence of electron screening on half-lives
}

\author{
G. Ruprecht;, L. Buchmann, D. Hutcheon, D. Ottewell, C. Ruiz, P. Walden, \\ C. Vockenhuber, K. Czerski ${ }^{+}$ \\ TRIUMF National Laboratory, 4004 Wesbrook Mall, Vancouver, BC, V6T 2A3, Canada \\ + Institute of Physics, University of Szczecin, Szczecin, Poland \\ E-mail: ruprechtatriumf.ca
}

Electron screening strongly changes nuclear reaction cross sections at energies below 1000 times the screening energy $U_{e}$. It has been found that $U_{e}$ can be one order of magnitude larger than predicted by theory [1] if the target atoms are hosted in a metallic environment. As a consequence, a change of lifetimes of low-energy $\beta$ and $\alpha$ emitters may also be considered if they are situated in a metal. In addition, a temperature dependence of the screening effect has been proposed [2], dramatically changing some half-lifes if the metal is cooled [3,5]. We checked these claims experimentally by measuring the decay rate of ${ }^{22} \mathrm{Na}$ in a piece of aluminum activated by a 70 $\mathrm{MeV}$ proton beam. We observed the ${ }^{22} \mathrm{Na}$ activity both at room temperature and when cooled down to nearly $\mathrm{LN}_{2}$ temperatures. As a result, a $10 \%$ increase as proposed by [3] can be clearly excluded. Furthermore, a $1 / \sqrt{T}$ temperature dependence of $U_{e}$ as it was proposed by [3,5] when the Debye-Hückel model is applied is unlikely.

International Symposium on Nuclear Astrophysics - Nuclei in the Cosmos - IX

June 25-30 2006

CERN, Geneva, Switzerland

\footnotetext{
* Speaker.
} 

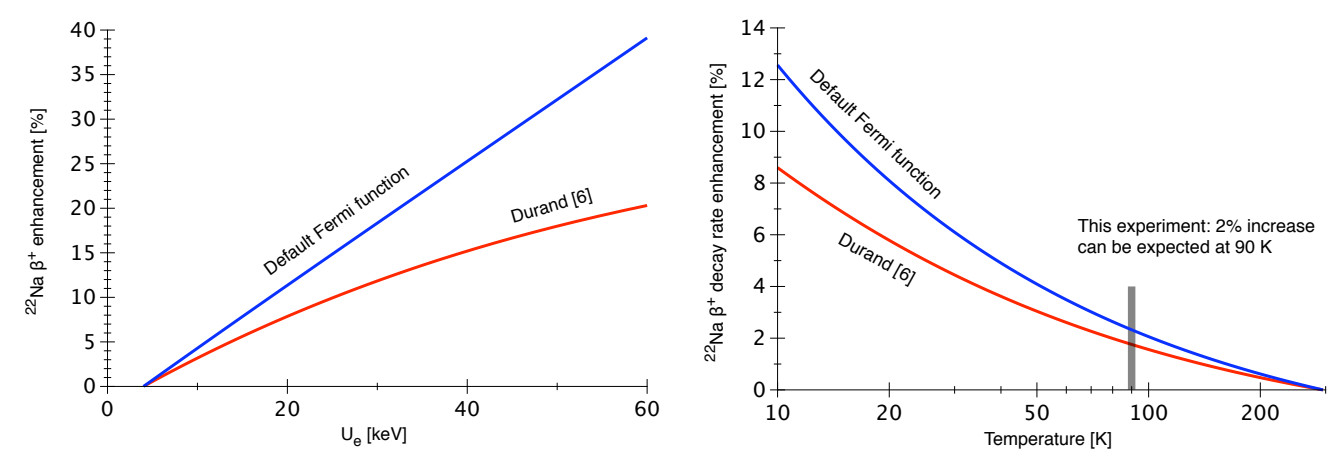

Figure 1: Left: Screening enhancement vs. screening energy $U_{e}$ for the ${ }^{22} \mathrm{Na} \beta^{+}$decay. The screening model of Durand [6] best describes the effect. Right: Enhancement of the ${ }^{22} \mathrm{Na} \beta^{+}$decay rate vs. temperature.

\section{Theory of the $\beta$ decay electron screening}

The number of end states per energy interval for the the $\beta$ decay can be described by a product of a phase space factor, the Coulomb penetration function $P(E)$, and the nuclear matrix element $|M|^{2}$ :

$$
\frac{d N}{d E} \sim\left(E+m c^{2}\right)(E-Q)^{2} \sqrt{E^{2}+2 E m c^{2}} \times P(E) \times|M|^{2},
$$

where $E$ is the kinetic energy of the emitted $\beta$ particle, $Q$ the Q-value of the decay, and $m$ the rest mass of the electron. In a simple picture, the electron screening can be described by an enhancement of the energy of the emitted $\beta$ by replacing $P(E)$ with $P\left(E \pm U_{e}\right)$ where $U_{e}$ is the screening energy and + is for $\beta^{+}$and - for $\beta^{-}$decay, respectively. Therefore, the decay rate is enhanced for the $\beta^{+}$decay and reduced for $\beta^{-}$decay. However, even for very low energies the $\beta$ particles must be treated relativistically and this simple picture of an "energy boost" fails. In a more detailed description [6] the Dirac or Klein-Gordon equation (if the spin plays no rule) must be solved for the Coulomb potential modified by the electron shell. This leads to the enhancement curves for the ${ }^{22} \mathrm{Na}$ decay shown in Fig. 1, left, for the $E_{\max }=Q=545-\mathrm{keV} \beta^{+}$transition to the $1274-\mathrm{keV}$ level of the ${ }^{22} \mathrm{Ne}$ daughter nucleus. The default Fermi function that is usually used for $\beta$ decay analyses can only be used for very small screening values.

\section{Temperature effect}

We are interested in possible temperature effects if the decaying $\beta^{+}$emitter is hosted in a metallic environment. If the screening energy $U_{e}$ scales with $1 / \sqrt{T}$ where $T$ is the absoulute temperature, the $\beta^{+}$decay rate should increase if the metal is cooled down. In the experiment (see below), ${ }^{22} \mathrm{Na}$ was hosted in Aluminum. The screening energy at room temperature can be estimated by multiplying the $\mathrm{d}+\mathrm{d}$ screening value for $\mathrm{Al}$ by the charge number of the ${ }^{22} \mathrm{Ne}$ daughter nucleus. Since there are large deviations for $U_{e}$ between [1] and [2] for $\mathrm{d}+\mathrm{d}$ screening we use a mean value of $400 \mathrm{eV}$ here.

The increase of the decay rate vs. the temperature is shown in Fig. 1, right. Since the absolute amount of implanted ${ }^{22} \mathrm{Na}$ is not very well known only changes at different temperatures can be 


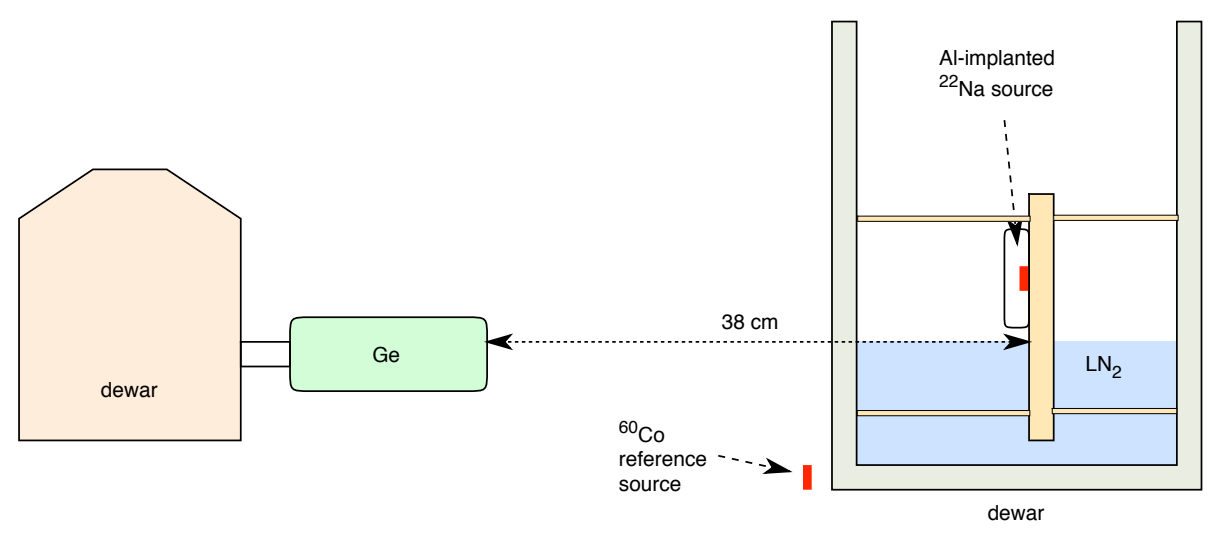

Figure 2: The set-up for the ${ }^{22} \mathrm{Na}$ lifetime measurement.

observed. Therefore, the curves are normalized to the enhancement at room temperature (295 K). About 2\% increase of the rate can be expected at $90 \mathrm{~K}$.

\section{The experiment}

The ${ }^{22} \mathrm{Na}$ source was produced at TRIUMF by sending a $70-\mathrm{MeV}$ proton beam through an aluminum disk. Via the reaction ${ }^{27} \mathrm{Al}\left(\mathrm{p},{ }^{6} \mathrm{Li}\right)$ most of the ${ }^{22} \mathrm{Na}$ is produced deep in the metal, as required by the claim in [3]. The source produced this way had an activity of $670 \mathrm{kBq}$.

The activated $\mathrm{Al} /{ }^{22} \mathrm{Na}$ probe was then mounted on a copper bar which was fixed with screwable rods at the walls of a dewar (see Fig.2). A ${ }^{60} \mathrm{Co}$ reference source was placed directly before the dewar. The $\gamma$-rays were detected with a Germanium detector. With a thermocouple mounted directly at the cover of the ${ }^{22} \mathrm{Na}$ source, the temperature could be determined. Measurements have beend done several times with and without $\mathrm{LN}_{2}$ in the dewar. The temperature at the source with $\mathrm{LN}_{2}$ filled in was about $90 \mathrm{~K}$. After these measurements the dewar was rotated by $180^{\circ}$ to exclude a change of the rate by a possible displacement of the source caused by mechanical stress and the measurements have been repeated.

\section{Results}

The results for both ${ }^{22} \mathrm{Na}$ lines are shown in Fig.3. When cooled to $90 \mathrm{~K}$, a small decrease of the 511-keV rate can be observed. However, similar fluctuations can also be seen at room temperature. The reason may be that $511-\mathrm{keV} \gamma$-rays can be produced by many other sources and are not unique to ${ }^{22} \mathrm{Na}$. (The measurements have been performed in the ISAC hall where many other experiments are running). However, the $1274-\mathrm{keV}$ line is clearly tied to our source. No correlation with the temperature can be seen for this line. If the 5 measurements at room temperature and the 3 measurements at $90 \mathrm{~K}$ are summarized each a small increase of $(0.70 \pm 0.45) \%$ can be observed. Therefore, an increase of $10 \%$ as it follows from a $40 \%$ increase at $10 \mathrm{~K}$ as claimed by [3] can be clearly excluded. Comparing this result with the expected enhancement at $90 \mathrm{~K}$ of $2 \%$ for the model by Durand and the non-relativistic screening enhancement, a visible effect is also unlikely (note that the default Fermi function is not suitable for high screening values as they are used here). 

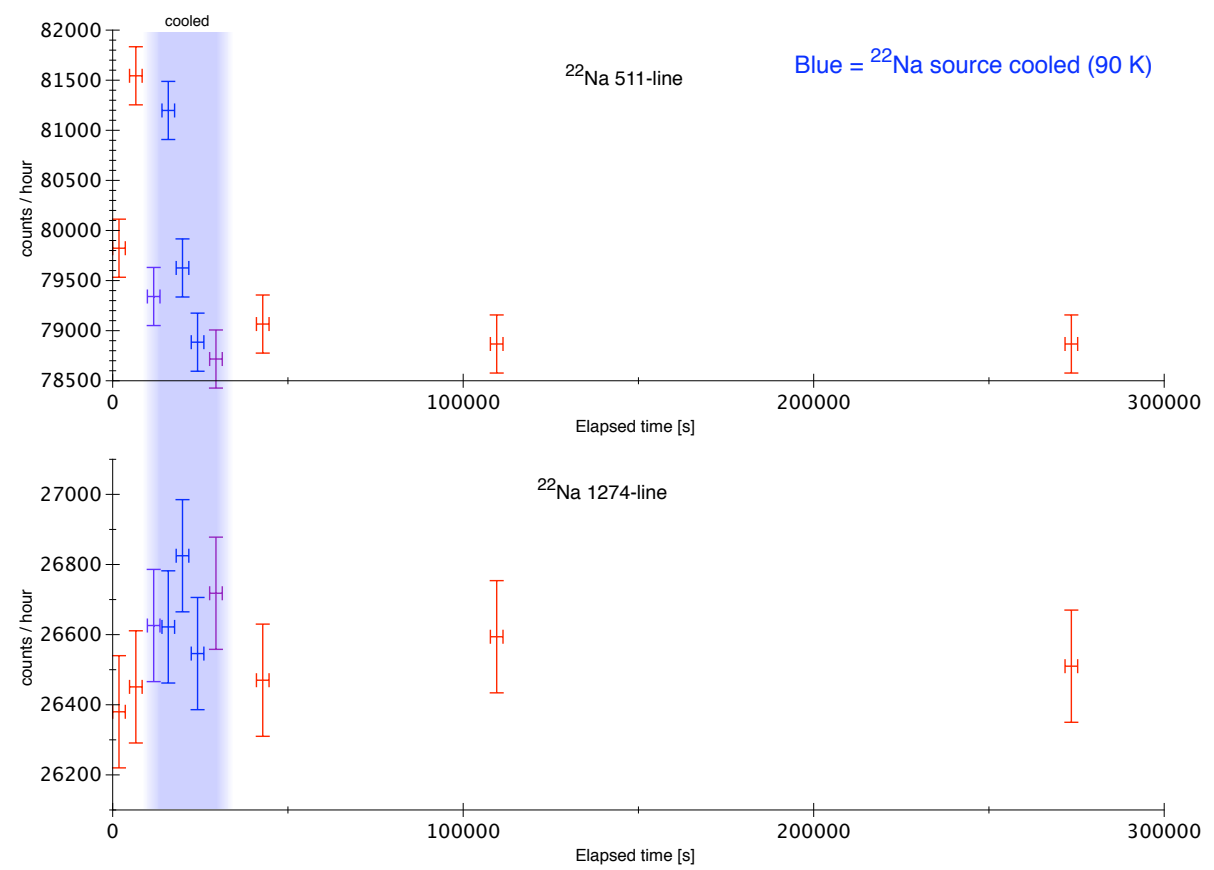

Figure 3: Results of the ${ }^{22} \mathrm{Na}$ lifetime measurement.

Concluding, high screening values at low temperatures as they result from a $1 / \sqrt{T}$ dependence as predicted by the Debye-Hückel model could not be observed within 3 standard deviations. The results are within 1.5 standard deviations in agreement with the expected Thomas-Fermi screening for dense electron plasmas predicting no temperature dependence.

\section{References}

[1] K. Czerski et al., Europ. Lett. 54(2001)449

[2] F. Raiola et al., J. Phys. G 31(2005)1141

[3] C. Rolfs, public lecture at SLENA 2006, Kolkata, India

[4] B. Limata, Eur. Phys. J. A 28(2006)251

[5] K. U. Kettner, et al., J. Phys. G 32(2006)489

[6] L. Durand et al., Phys. Rev. 135(1964)B310 